\title{
Trajectoires de vie et rapports de domination.
}

\author{
Des travailleuses migrantes à Buenos Aires
}

\section{Natacha Borgeaud-Garciandía}

\section{Résumé}

Cet article prend appui sur des récits d'employées domestiques et du care à Buenos Aires. Leurs trajectoires sont marquées par la migration, tandis que la place occupée dans la division du travail en Argentine témoigne de l'articulation de divers rapports de domination (sexe, 'race', classe). De là, l'analyse porte sur la complexité de ces rapports, tels qu'ils se structurent dans les récits que les sujets offrent de leurs trajectoires de vie. Une première partie situe la problématique dans une conceptualisation des rapports entre dominations et sujets. Une deuxième partie restitue de façon analytique une trajectoire, à même d'illustrer et de mettre en lumière la complexité et la variabilité des rapports entremêlés de la domination sur l'échelle d'une biographie et des représentations auxquelles elle donne lieu. ${ }^{1}$

EMPLOYÉES DOMESTIQUES — CARE — ARGENTINE — DOMINATION — FEMMES ET MIGRATIONS

L'un des enjeux de la littérature sur les migrations féminines de travail consiste à évaluer si celles-ci doivent être envisagées sous l'angle de la domination ou de l'émancipation, tandis que des recherches récentes travaillent au contraire à en montrer

\footnotetext{
${ }^{1}$ Je remercie sincèrement Danièle Senotier pour son sérieux et sa prévenance. Ma reconnaissance va également aux évaluateurs/évaluatrices de la revue dont les commentaires m'ont permis d'améliorer sensiblement ce texte.
} 
l'ambiguité ${ }^{2}$. Dans le même esprit, et dans la continuité de nos travaux précédents, la réflexion est ici centrée sur les jeux et enjeux des rapports de domination sur le cours de trajectoires individuelles. L'attention porte non seulement sur ce que les rapports de domination font aux trajectoires mais, à l'inverse, sur ce que ces dernières font et nous disent des rapports de domination. À partir des récits de vie de travailleuses du care migrantes en Argentine et de la reconstitution subjective de leurs parcours, nous nous pencherons sur la complexité des dominations et de leurs entrelacements le long des trajectoires - dans le cas présent de femmes originaires du Pérou et du Paraguay, qui travaillent comme employées domestiques ou auprès de personnes âgées dans la ville de Buenos Aires.

En Argentine, on distingue deux grands ensembles de courants migratoires très différemment perçus par la population locale (Benencia 2004). L'image des migrations européennes « civilisatrices » (Pacecca 2009) (fin XIX ${ }^{\mathrm{e}}$-mi XX ${ }^{\mathrm{e}}$ siècle) contraste avec les représentations qui affublent le second courant migratoire composé de travailleurs et (de plus en plus) de travailleuses issu·e·s des pays de la région latino-américaine. Malgré la stabilité numérique des migrations régionales ${ }^{3}$, elles ont connu d'importantes transformations internes, comme par exemple leur composition suivant leur origine ou encore leur point de chute. Parmi ces transformations, leur féminisation représente un changement essentiel. Visible dès les années 1960 dans les migrations paraguayennes, elle est tardive mais exponentielle dans le cas des Péruviennes, et moins importante pour les populations boliviennes, dont les déplacements et les activités sont davantage familiaux-communautaires (Crivelli 2014) ${ }^{4}$.

\footnotetext{
${ }^{2}$ Pour des analyses critiques d'une approche duale en termes de domination $v s$ émancipation, cf. Attias-Donfut et Delcroix (2004), Moujoud (2008), de Montvalon (2013) et Zeneidi (2013).

${ }^{3}$ Le pourcentage tourne autour de $3 \%$ de la population totale depuis le début $\mathrm{du} \mathrm{XX}^{\mathrm{e}}$ siècle.

${ }^{4}$ Concernant ces trois flux migratoires, le taux de masculinité est passé de 117, 5 hommes pour 100 femmes en 1980 à 83 hommes pour 100 femmes en 2001 (Courtis, Pacecca 2008). Le dernier recensement (2010) a pour le moment fait l'objet de moins d'études. Certaines d'entre elles - la plupart - mettent en avant la composante majoritairement féminine de ces flux (Calvelo 2012),
} 
Plus la féminisation de ces migrations est importante ${ }^{5}$, plus leur concentration est élevée dans l'Aire métropolitaine de Buenos Aires ${ }^{6}$, et dans les services domestiques et du care (Pacecca 2009). La migration issue du Pérou est la plus éloquente : au cours des années 1990, elle représente $40 \%$ des flux en provenance des pays cités, et $67 \%$ de ces migrant·e's péruvien.ne's entre 35 et 59 ans sont des femmes (Cerrruti 2005). Finalement, en Argentine :

La grande majorité des Péruviennes et des Paraguayennes travaille dans le secteur des services à la personne (respectivement $69 \%$ et 58,1\% [en 2001$]^{7}$ ). Cette concentration dans l'une des activités les plus marquées par des rapports d'exploitation [...] est l'une des plus élevée de la région, et probablement du monde. Une situation qui contraste avec la proportion bien plus faible de femmes argentines employées dans les services domestiques (15,7 \%) (Cerruti 2009).

Traditionnellement occupées par les migrantes internes, ces activités se transforment en emplois-refuges pour les migrantes latino-américaines ${ }^{8}$ au point que presque une employée sur deux dans la ville de Buenos Aires est issue de ces migrations.

Nous avons rencontré, au cours de notre recherche, des cuidadoras (aides à domicile pour personnes âgées) employées dans la capitale argentine. Parmi les migrantes péruviennes, la

tandis que d'autres soulignent que ces dernières années témoignent d'une tendance légèrement à la baisse ( $c f$. note suivante).

${ }^{5} \mathrm{La}$ féminisation de la migration connaîtrait depuis quelques années une baisse, peut-être due aux effets de la reprise économique qui aurait profité aux emplois essentiellement masculins, notamment la construction qui concentre de la main-d'œuvre migrante masculine (Castillo, Gurrieri 2012).

${ }^{6}$ L'AMBA comprend la ville de Buenos Aires et les vingt-quatre municipalités qui l'entourent. Elle concentre $30 \%$ de la population totale du pays.

${ }^{7}$ Ces chiffres varient selon les sources ( $c f$. Pacecca 2009), mais sont toujours très élevés.

${ }^{8}$ Soit que les migrantes internes désertent ces emplois (Pacceca 2009), soit qu'elles se font concurrence, les secondes acceptant des conditions de travail plus précaires que les premières (Cortés, Groisman 2004), à moins que la concurrence ne soit réapparue en période de crise économique et sociale (fin 1990-début 2000). La concurrence existe également entre les migrantes, les Péruviennes ont déplacé les Paraguayennes notamment du fait de leur niveau scolaire plus élevé pour les mêmes emplois (Courtis, Pacceca 2010). 
plupart sont arrivées en Argentine dans les années 1990, poussées par la crise économique et sociale ainsi que la violence ${ }^{9}$ qui minait leur pays, et attirées par la proximité, la langue, la possibilité de travailler, le taux de change qui permettait d'envoyer de l'argent à la famille restée au pays. Elles sont arrivées seules, suivant les conseils d'une compatriote, amie ou de la famille, travaillant en Argentine et leur ayant parfois déjà procuré un emploi dans un foyer argentin. Une fois stabilisées en Argentine, plusieurs d'entre elles ont fait venir maris et enfants. Sur place, c'est à quelques exceptions près dans les secteurs domestiques et du care qu'elles trouvent un emploi, non seulement à domicile mais souvent 'à demeure' (interne). Ce sont sans surprise des activités caractérisées par la grande précarité des conditions d'emploi et de travail ${ }^{10}$. Toutes ont réalisé des études secondaires, tertiaires ou supérieures dans leur pays, et leurs connaissances (infirmerie, anglais, formation d'institutrice) ne manqueront pas d'être exploitées par leurs employeurs; toutefois leurs trajectoires de travail demeurent assujetties à des niches modelées par des critères entrecroisant le genre et l'appartenance d'origine, la classe ou la migration (celle-ci surplombant celle-là).

C'est justement sur les entrelacements complexes entre dominations (pré et post migration) et trajectoires de vie que nous souhaitons réfléchir, partant des récits de vie collectés auprès des travailleuses rencontrées, et que nous illustrerons ici plus particulièrement avec le parcours d'Olga. Cette réflexion naît de la difficulté qui nous est apparue à l'heure d'analyser les reconstructions de la trajectoire propre dans les discours singuliers. Celle-ci ne peut pas se réduire à la place que les migrantes occupent dans la division du travail en Argentine. Comme le rappelle Yannick Le Quentrec (2009, p. 219), «les rapports sociaux de domination, de classe de 'race', et de sexe ne font

\footnotetext{
${ }^{9}$ Les années de profonde crise économique au Pérou sont aussi celles de la violence extrême qui oppose la guérilla du Sentier lumineux aux militaires et paramilitaires (cf. Cerruti 2005).

${ }^{10}$ La précarisation du travail touche encore plus les femmes migrantes que le reste de la population. Par exemple, le travail illégal touchait $73,8 \%$ de cette population en 2003 contre une moyenne de $45 \%$ pour l'ensemble de la population (Cacopardo 2005).
} 
pas disparaître la puissance d'agir des individus en vue de leur émancipation ». Ceux-ci, en tant que sujets, imposent des écarts, des ruses, des ouvertures - de même que des dérivatifs et des défenses assujetissants - aux rapports précités et aux places par eux assignées ${ }^{11}$.

Dans cet esprit, la première partie de l'article situe son analyse par rapport aux recherches qualitatives que nous avons menées précédemment auprès d'ouvrières de maquiladoras : il est rapidement apparu qu'il importait de prendre en compte 'l'entièreté de la vie' des enquêtées (analyse synchronique et diachronique) (Borgeaud-Garciandía 2008a). Les cuidadoras ajoutent un élément important : la migration, qui sera saisie partant de la reconstruction subjective qui est faite de la trajectoire à la fois personnelle, professionnelle et familiale. De là nous cherchons, dans la deuxième partie, à montrer à travers la trajectoire d'Olga enrichie d'éléments issus d'autres trajectoires, des jeux, des décalages et des transformations qui travaillent les rapports sociaux de domination sur le temps long de la biographie. Une synthèse analytique sur les relations entre trajectoire, rapports de domination et sujets clôt cette approche par la trajectoire (troisième partie), et ouvre sur la conclusion.

\section{Centralité du travail et récits biographiques}

Nos recherches actuelles portent sur les dominations dans et par le travail et la mise au travail ${ }^{12}$, partant de récits biogra-

\footnotetext{
${ }^{11}$ Nous ne nous situons pas sur le plan de 'l'émancipation' à proprement parler, mais 'en-deçà', du côté des potentialités, des possibles compris dans les écarts, les décalages qui peuvent relever de puissances vitales ou de stratégies défensives. La trajectoire présentée est choisie en fonction de sa capacité à illustrer et à organiser la réflexion ; elle renvoie également à d'autres trajectoires qui ne sont pas nécessairement passées par des engagements collectifs.

${ }^{12}$ Ce terme revoie d'une part au fait même de 'mettre au travail' certaines catégories de la population; d'autre part, aux modalités que cela implique. Dans les cas analysés, celles-ci sont diverses et se situent souvent à cheval entre l'emploi 'formel' et 'informel'. Elles vont de formes de salarisation au rabais (dans le cas des maquiladoras nicaraguayennes), dont la précarité n'a pas forcément à envier à certaines formes d'emploi dans l'économie informelle à, justement, des emplois 'indépendants' (dans lesquelles la relation
} 
phiques. Les expériences de travail y sont centrales - suivant l'hypothèse selon laquelle le travail (en soi, dans sa division, dans ses effets) est à la fois central pour la construction du sujet et comme vecteur de domination. Ainsi, dans l'analyse des récits de vie offerts par des ouvrières des maquiladoras, il est apparu que les contraintes liées au travail et à la mise au travail s'étendent à l'organisation de la vie entière des ouvrières, saisissent leurs temporalités, pénètrent leur vie jusqu'aux recoins les plus intimes, laquelle, à son tour, se structure et s'articule autour de ces exigences (Borgeaud-Garciandía 2009).

Notre approche a ainsi porté sur les rapports complexes, ambigus des sujets à la domination qui s'exerce par le truchement de la mise au travail et des contraintes qui lui sont liées. Nous ne nous plaçons ni du côté d'une domination 'aveuglante' interdisant toute prise de distance de la part des sujets, ni d'un acteur complètement lucide capable d'en décrypter les trames. Très schématiquement, il apparaît que les contraintes ne sont pas que limitatives, elles sont productives. Mais elles ne produisent pas des 'sujets de la domination' à proprement parler. Dans l'auto-affirmation (qui peut être défensive ou idéalisée) d'un sujet par le récit de sa propre histoire, s'impose un soi qui ne se réduit pas aux places assignées par les relations de pouvoir. Dans la recherche de cohérence et de production d'un soi malgré les contraintes limitatives, apparaît un sujet en décalage, potentiellement imprévisible, un sujet qui se crée à la fois avec, par et contre la domination. Ce qui retient notre attention, c'est la production du sujet travailleur - femme et homme - par luimême en milieu contraint ; sujet non dénué de capacité d'action qui, par la prise de parole, se constitue à la fois 'en marge', voire 'contre' la domination, cependant qu'il ne peut se constituer autrement qu'avec' elle, la reconnaissant et répondant à ses exigences (Borgeaud-Garciandía 2009).

Si dans le cas des ouvrières des maquilas, le travail et ses exigences envahissent la vie entière (Borgeaud-Garciandía 2012a), dans le cas des cuidadoras migrantes travaillant à demeure, la vie au présent est subsumée par la vie au travail. Nos analyses

d'emploi n'est pas reconnue) ou illégaux dans le cas des cuidadoras en Argentine. 
ont alors porté sur les rapports au care effectué dans des conditions particulièrement difficiles, où de nouveaux facteurs doivent être pris en compte dans l'analyse des rapports du sujet au travail, tels que la cohabitation permanente avec le sujet dépendant, la sénilité et le corps qui faiblit, l'enfermement, ou encore le rôle que jouent les affects, la responsabilité, ou l'éthique dans la possibilité de demeurer auprès d'une vieille personne malade sans y laisser sa propre raison (Borgeaud-Garciandía 2012b, 2014).

Or, outre les aspects proprement liés au travail, la migration signifie bien souvent un bouleversement majeur dans le cours de la vie (même par défaut), une ouverture sur des possibles, et joue sur la perception des trajectoires et les formes de réinterprétations auxquelles elles donnent lieu (Borgeaud-Garciandía 2008b). En ce sens :

La reconstruction d'un parcours biographique ne peut aucunement être confondue avec la trajectoire objective de celui qui, répondant à la sollicitation du chercheur, se raconte (Galloro, Pascutto, Serré 2010).

Chaque enquêtée est, au cours de l'entretien, partie de là où elle le souhaitait, enfance pour certaines, arrivée en Argentine pour d'autres ou encore présent immédiat, puis a suivi les méandres de ses expériences comme elle désirait les transmettre. Olga présente d'emblée la fillette battue dont elle estime avoir changé le cours du destin, tandis que le récit d'Estrella débute avec la migration et il faudra une intervention de la chercheuse pour que ses cinquante premières années de vie soient racontées. Comme le signalent Galloro, Pascutto et Serré (2000, p. 18), "saisir les éléments qui influencent le déroulement d'une mise en récit est d'une grande complexité », notamment du fait de la place occupée par le sujet au moment de son récit. Dans leur recherche, ces auteurs montrent ainsi que les récits diffèrent entre les travailleurs algériens restés en France et ceux retournés en Algérie. Dans nos propres entretiens, nous observons des variations notamment en fonction du type d'emploi occupé (schématiquement entre les personnes qui réalisent de multiples activités et les cuidadoras à demeure sujettes à un temps continuellement 'au présent', envahissant, qui entrave projections et prises de distance). Ces variations dans la présentation des trajectoires ne peuvent être analysées ici. Gardons simplement à 
l'esprit que récits et trajectoires sont saisis en tant que construits, et que la migration affecte aussi bien le regard porté sur son histoire que la situation présente et les perspectives futures, notamment du point de vue des vécus saisis par des enjeux de domination ${ }^{13}$.

\section{Trajectoire et récit d'Olga}

Les analyses de la domination qui le mieux articulent travailmise au travail et migrations féminines (généralement des pays du Sud vers ceux du Nord), croisent à la fois les rapports de sexe/genre, de race/ethnie/nationalité, et de classe (Falquet et al. $2006,2008,2010)$. Ce n'est en effet pas sans raison que la plupart des migrantes latino-américaines (mais là encore plutôt les Paraguayennes et les Péruviennes que les Uruguayennes et les Brésiliennes) se retrouvent dans les secteurs de domesticité et du care, et qu'à l'inverse ces emplois semblent réservés à des employées femmes, pauvres ou migrantes. Si ces analyses ne représentent pas une nouveauté absolue en France (Falquet, Lada, Rabaud 2006), elles font depuis quelques années, notamment sous l'impulsion des théories de l'intersectionnalité, l'objet d'un intérêt croissant. Elles ont pour grand avantage de décloisonner les catégories en reconnaissant l'hétérogénéité du social «qui n'est pas le produit d'un seul rapport social mais de plusieurs » (Pfefferkorn 2012, p. 123), qui sont à la fois coextensifs et consubstantiels (Kergoat 2009). Les analyses multidimensionnelles mettent en outre l'accent sur l'articulation des dimensions objectives et subjectives, ainsi que la production et la reproduction des rapports sociaux à la fois comme source de domination et d'émancipation potentielle (Kergoat, Miranda, Ouali 2011, Pfefferkorn 2012). Le travail (productif et reproductif, son organisation, ses divisions) demeure central dans la production des rapports sociaux et en tant que levier de la domination. Il n'est pas surprenant que le travail du care, notamment en ce qu'il affecte un nombre croissant de femmes migrantes issues de pays pauvres, représente un exemple paradigmatique de la production mutuelle de ces rapports sociaux (Kergoat 2009).

${ }^{13}$ Cf. l'analyse particulièrement illustrative réalisée par Djemila Zeneidi (2013). 
Dans la continuité de nos analyses précédentes, plutôt que les places (mouvantes) assignées par ces rapports sociaux (mouvants), ce sont les écarts, les ouvertures, les retournements, ou encore les reproductions et les renforcements que les individualités leur imprègnent qui nous intéressent, et qui introduisent une perspective heuristique à la croisée des rapports de classe, 'race', sexe et des trajectoires et perceptions. Les trajectoires analysées sont marquées par une multiplicité de positions occupées, aux configurations changeantes, qui plus est dans un contexte marqué par la migration de nature à entraîner un bouleversement des positionnements sociaux des acteurs et actrices. Nous avons choisi d'illustrer les variations et dissonances des rapports de domination qui pèsent sur les parcours et représentations partant de la reconstruction d'une trajectoire, celle d'Olga, qui sera également appuyée par d'autres exemples.

La trajectoire d'Olga, travailleuse migrante péruvienne, n'est en soi ni banale, ni exceptionnelle, elle a toutefois l'avantage de présenter de manière particulièrement suggestive et accentuée les méandres de son parcours. Ce choix peut être aussi lié à cette réponse lâchée au cours de l'une de nos rencontres. Lorsque je lui demande si elle ferait ce même travail au Pérou — « le travail auprès des abuelas [mamies], le travail domestique, tu veux dire ? », elle secoue négativement la tête :

Non. Là-bas, nous sommes très discriminateurs. Parce que nous sommes discriminateurs. Et par orgueil. Que te dire? Non, je ne le ferais pas. Je ne le ferais pas.

L'image prototypique et très négative de l'employée domestique au Pérou est celle de la jeune ou petite fille indienne pauvre et sans éducation, quechua parlant mal l'espagnol, ayant migré des zones montagneuses pour travailler en ville (Figueroa 2007). Le point de vue partagé par Olga, employée domestique à Buenos Aires au moment de notre rencontre, témoigne de points de rencontre et de négociation entre son vécu et les positions sociales telles qu'elles se structurent 'ici' et 'là-bas'. Il est impossible 'par orgueil' de s'identifier à celles qui exercent ce métier-là au Pérou. Bien que son statut en Argentine soit débiteur de la place réservée aux migrantes latino-américaines par le truchement de la division sociale 'racialisée' du travail, il 
demeure acceptable, tandis qu'une position analogue serait inconcevable dans son pays d'origine.

Il est possible, à des fin d'analyse et d'exposition, de distinguer quatre grandes étapes dans la trajectoire d'Olga au cours desquelles sa vie familiale, professionnelle et personnelle a été bouleversée.

\section{Violences infantile et domestique.}

De la négation à la conscience de soi

La première image qu'elle donne d'elle-même, pour mieux s'en distinguer par la suite, est celle de la petite fille de huit ans, envoyée par sa mère travailler comme bonne ou nanny d'enfants encore plus jeunes afin d'échapper aux coups que lui inflige son beau-père. Orpheline à l'âge de 12 ans, elle vit ensuite avec son oncle aimé jusqu'au départ de celui-ci à Lima où elle refuse de l'accompagner. Restée seule à Chimbote, ville côtière et port de pêche dont elle est originaire, elle rencontre à l'âge de 15 ans un garçon. Elle aura avec lui, pêcheur de son métier qui dépense son salaire en alcool, sept enfants dont trois meurent de maladies, deux en bas âge et une à six ans. Olga prend des pastilles pour dormir, vit avec ses enfants sans pratiquement sortir de la maison, dans la crainte de ce mari ivrogne. Elle dira - alors même, qu'enfant, elle reprochait à sa mère de ne pas réagir aux coups infligés par son propre mari - qu'une femme ne prend conscience de la violence subie qu'une fois qu'elle en est sortie. Un jour, invitée par une amie, elle décide - contre l'avis de son mari qui lui reproche violemment d'avoir davantage d'étude et de formation que lui ${ }^{14}$ - de suivre un cours de coupe et couture, "pour sortir de chez moi ». Puis, elle commence à prendre en cachette des pilules contraceptives. Le mari les découvre, l'accuse d'adultère et lui assène un coup qui lui endommage le tympan. C'est le coup de trop. Elle profite que la pêche l'appelle longuement hors de la ville pour cacher ses affaires et porter plainte pour abandon du domicile conjugal. Supplications et promesses de changement n'y feront rien, Olga ne reviendra jamais sur sa décision. Une première page est

\footnotetext{
${ }^{14} \mathrm{C}$ 'est une fois adulte qu'Olga achèvera ses études secondaires et suivra des formations (voir plus loin).
} 
tournée. Elle n'a pas d'argent, pas de travail, quatre enfants à sa charge, mais, dit-elle, "c'est alors que j'ai commencé à vivre ».

Les souvenirs d'enfance et de jeunes femmes au Pérou varient en fonction des histoires individuelles, de l'époque à laquelle il est fait référence, mais portent souvent des traces de séparations, entre enfants et parents, entre jeunes femmes et conjoints. Une autre cuidadora, Estrella, dira de son premier mari qui la confinait à l'espace du foyer (elle avait notamment dû refuser une bourse qui lui aurait permis d'achever sa formation d'infirmière aux États-Unis) :

Je n'ai pas souffert de me séparer [...]. J'étais une enfant de plus et pas une femme [...]. [Je n'étais là] que pour avoir des enfants, comme si le fait d'avoir beaucoup d'enfants pouvait me retenir là, prisonnière, mais ce n'est pas comme ça. On peut avoir vingt mille enfants, arrive un moment où l'on dit « basta », on rompt les chaînes et c'est fini.

Elle paiera cette décision au prix cher : son premier mari lui retire un à un ses enfants qu'elle ne retrouvera que devenus adultes.

La première image qu'Olga donne d'elle-même n'est pas seulement celle de la petite fille sans protection, de toute part assujettie, c'est aussi celle de la fillette (certes devenue adulte) offensée, dépourvue de pouvoir mais qui porte un regard sans condescendance sur son entourage. Ce n'est que plus tard, lorsqu'elle vivra coupée du monde dans la crainte et sous les coups de son mari, qu'elle s'est sentie dépossédée d'elle-même, amputée de toute distance par rapport à sa propre situation. Néanmoins, elle étouffe et peu à peu, le désir s'immisce, les rouages grincent. Ce qui l'assujettit ne suffit plus à la contenir, Olga advient par excès sur la place assignée. Non point affranchie des entraves mais la conscience claire, elle œuvre à dégager une sortie. Des plus fragiles et précaires, mais une sortie.

\section{Engagements et capacité d'agir}

J'avais vu une autre vie. Je savais que je pouvais travailler et nourrir mes enfants.

C'est, pour la jeune Olga de 26 ans, une découverte d'ellemême, un renouveau. Elle fait de tout, offre ses services pour réaliser des démarches administratives à la municipalité, achète 
et vend des vêtements «Je suis sortie » hors de l'espace du foyer. Parallèlement à cela, elle croise à nouveau le chemin d'un amour de jeunesse qui deviendra son deuxième mari et le père de ses deux derniers enfants. Il l'aide et lui apporte son soutien à chacun de ses nombreux projets. Elle reprend et achève ses études secondaires avant de suivre une formation en secrétariat.

Depuis que je suis 'sortie de lui' [premier mari], j'ai fait beaucoup de choses.

Elle découvre la politique «au passage», tisse des liens, construit ses réseaux; elle devient cadre militante du Parti Apriste péruvien ${ }^{15}$. Entre temps, elle obtient un petit contrat à la municipalité, gagne un concours d'institutrice (métier qu'elle n'aime pas), puis décroche un poste à la municipalité quand le représentant du parti Apriste qu'elle soutient remporte les élections locales. Dans un premier temps, elle s'occupe de l'organisation des nombreuses fêtes patronales, ces festivités d'origine plus ou moins religieuse qui ponctuent les douze mois de l'année. C'est la seule femme à occuper ce poste dans le pays. Il faut batailler avec les forains qui disent d'elle qu'elle est «pire que les hommes ». Puis elle travaille quelques années comme secrétaire de direction dans une entreprise de recouvrement montée et sous-traitée par la municipalité, avant de retourner à la mairie travailler, au niveau du district, comme coordinatrice générale d'un programme national de distribution de lait aux enfants. Là aussi, il faut batailler pour ne pas se faire marcher dessus. Olga poursuit ses activités militantes, elle est élue présidente de la commission départementale pour le «Noël des enfants » du parti Apriste, où elle travaille également comme secrétaire du conseil de discipline.

Je n'étais plus la petite fille battue qui travaillait depuis ses huit ans ; j'étais [devenue] une autre personne.

Son mari quitte son emploi dans une industrie sidérurgique et achète une camionnette pour réaliser du transport de passagers

\footnotetext{
${ }^{15}$ Né en 1930, le PPA représente l'une des plus importantes organisations politiques au niveau national. Il est issu de l'Alliance populaire révolutionnaire latino-américaine (APRA), mouvement péruvien anti-impérialiste à visée continentale fondé en 1920 .
} 
interurbain. En 1994 arrive un nouveau maire, issu d'une autre fraction du parti Apriste, à la tête de la municipalité. D'après Olga, il est prêt à la garder à condition qu'elle le lui demande ; et ce serait à son refus qu'elle doit de se trouver en Argentine. Pourtant, les déboires postérieurs précipitent la décision. Tous deux travaillent avec la camionnette. Tandis que le mari conduit, elle crie les destinations et encaisse - elle est l'une des premières femmes à effectuer ce travail. Mais le moteur tombe en panne et avec lui leur gagne-pain. Nous sommes en 1997. D'Argentine, une copine lui propose de la rejoindre. De son départ, elle ne peut oublier l'image de son fils de trois ans, endormi, qu'elle s'apprête à quitter.

Si la débrouille peut être associée à l'absence de choix de personnes plongées dans les difficultés économiques, elle est ici présentée par Olga comme une ouverture des possibles. Olga, enserrée par nombre de contraintes, sociales et pratiques, offre d'elle l'image d'une femme qui peut enfin tracer son chemin, qui commence à rompre avec des années d'une dépendance conjugale qui la coupait du monde. Elle se teste, elle s'essaie, elle se découvre. Et se présente comme l'actrice principale de ce bout de destin où s'articulent inextricablement dans son récit l'amour retrouvé et reconquis, l'ascension à la fois professionnelle et militante. Les difficultés et la pesanteur des contraintes sociales, dont on perçoit tantôt les entraves (n'oublions pas qu'elle est femme dans des univers masculins, mère de famille et dépourvue, les premiers temps du moins, de soutien familial et de réseaux structurés), sont minimisées au profit d'une capacité d'agir dont Olga n'est pas peu fière. Pourtant les contraintes économiques rattraperont la famille. Le discours d'Olga a changé de registre et elle se refuse à présenter la situation sous l'angle de la fatalité. C'est encore elle qui prendra la décision de partir, comme beaucoup d'autres femmes, sans l'aval convaincu de la famille.

\section{Migration et mise au travail. Entre contraintes et élaboration de repères}

La migration n'est pas présentée comme une fatalité absolue. Les migrantes préfèrent, rétrospectivement du moins, l'inscrire dans un projet, prévu pour être temporaire puis qui s'est indéfi- 
niment prolongé. Acculées, pourtant, par les conséquences de la crise économique (Olga, Estrella, Rosalba ne s'en sortent plus financièrement, Lucía est criblée de dettes), elles présentent la migration comme relevant d'une décision à la fois personnelle et fruit de compromis familiaux. Ceux-ci peuvent même se traduire par une «division familiale des migrations de travail féminines »: Rosalba et Oriana sont venues travailler en Argentine tandis que leurs filles, dont certaines sont également cuidadoras, ont migré vers l'Espagne et les États-Unis. Pour Olga, ce départ, lié aux circonstances économiques familiales et sociales, c'est un peu la concrétisation d'un vieux rêve :

J'ai toujours voulu venir. Déjà dans les années 1980. J'étais déjà avec mon mari mais je voulais venir. Mon rêve était toujours de partir du pays, non pas par nécessité mais pour connaitre, comme ceux qui désirent partir comme on désire voler.

Pourtant, le voyage, son arrivée et les premiers temps en Argentine sont extrêmement difficiles. Seule, éloignée de sa maison et des siens, elle doit s'entasser avec cinq autres personnes dans une chambre de pension et travailler pour rembourser le montant du billet à son amie. Les récits que les migrantes offrent des premiers mois en Argentine traduisent une grande pénibilité. Lucía connaîtra les pensions, les squats sans électricité ni eau, la tuberculose ; Celia circulera entre les pensions, les logements des employeurs, la rue et les squats; Estrella, logée dans un bidonville, dormira sur un matelas posé à même le sol dans l'unique pièce commune; d'autres se retrouvent d'un coup chez des employeurs dont elles ne connaissent absolument rien.

Sommée par son amie de trouver du travail et de la rembourser aussitôt, Olga trouve un emploi de domestique à demeure dans un quartier résidentiel fermé ; les fins de semaine, elle fait des ménages et propose aux résidents de la pension de laver leur linge. Elle parvient à payer sa dette et à envoyer un peu d'argent à son mari. Peu à peu, elle s'organise. Elle quitte son premier emploi suite aux diverses accusations de sa patronne (utilisation abusive de papier toilette, mauvais emploi de la machine à laver, etc.). Elle est tour à tour employée pour le ménage et le service dans une chocolaterie, puis pour un temps limité comme employée domestique chez une vieille dame et, pour la première fois, comme cuidadora chez un vieux monsieur. En parallèle, 
elle distribue des tracts et des publicités, ou revend des boissons aux locataires de la pension. Logée ou pas chez l'employeur, elle garde la chambre de pension dans laquelle elle est maintenant seule car elle prévoit de faire venir son mari et son plus jeune fils. Ils migreront effectivement à leur tour, alors qu'une compatriote lui a laissé sa place de cuidadora à demeure auprès d'une vieille Italienne à laquelle elle s'attache beaucoup et dont elle prendra soin jour et nuit pendant six ans - jusqu'à ce que sa fille, employeuse d'Olga, décide de placer sa mère en institution sans préavis et sans aucun geste de reconnaissance. Tout comme une grande partie de ses compatriotes (femmes), Olga travaillera encore plusieurs années dans le care aux personnes âgées, parfois à l'heure, parfois à demeure, par l'intermédiaire de ses réseaux ou de ceux de ses employeurs. Son mari travaillera quant à lui d'abord dans la construction, avant d'être employé comme gardien de la pension où ils habitent. Toutes les migrantes ne font pas venir leur famille, et il est certain que cette décision a un impact essentiel sur le déroulement de la trajectoire professionnelle et sociale qui s'ensuit (travail de care à demeure ou emplois rémunérés à l'heure).

Pour Olga comme pour d'autres, il est admis que les emplois du care sont dévolus aux migrantes, sur la base de la naturalisation et la généralisation de qualités affectives, et d'une différenciation nous/eux, nous/les autres, et là-bas/ici. Ainsi, pour elle :

Ils cherchent surtout des Péruviennes pour travailler auprès des abuelos [papis-mamies] [...] parce que la femme péruvienne a beaucoup plus d'amour pour ses vieux, [...] plus de patience, plus de tendresse pour leurs vieux. Nous, nos vieux, on ne les abandonne pas.

Elle ajoute plus loin :

Je suis venue parce qu'il y avait du travail. [...] Les hommes et les femmes péruviens sont très travailleurs [...]. C'est, peut-être, une différence avec les autres communautés.

Les stéréotypes mobilisés (peut-être dans le but de légitimer la place échue) ne sont pas une simple reproduction de ceux qui, en Argentine, assignent les migrantes à ces emplois. Ils leur font écho et les renforcent, tout en prenant appui sur des différenciations avec les Argentin'e·s et entre migrant·e·s. 
Ce jeu entre stéréotypes et assignations identitaires, Celia - en couple avec un Bolivien, cuidadora devenue entrepreneuse, évangélique - veut les déjouer. Une Péruvienne et un Bolivien 1) à la tête d'une entreprise, 2) qui n'est pas une fruiterie (autre niche ethnique), 3) qui plus est, avec à sa direction une femme : "beaucoup de gens se surprennent" (ils ne sont pas là où on les attend, en particulier elle). Pour elle, les stéréotypes - ainsi résumés "Péruvien, feignant, profiteur, voleur ; Bolivien, travailleur, soumis ; Paraguayen, travailleur, oiseux, brutal ou ivrogne » - ont la peau dure dans la société argentine. De ces stéréotypes que l'on entend parfois, les femmes semblent quasi absentes, comme si leur confinement dans l'espace domestique les exonérait en partie du poids de ces stigmates, tout en renforçant ceux qui les attachent aux emplois qu'elles occupent.

Les travailleuses en prise avec les difficultés que réserve la situation de migrantes sont en effet confrontées à des rapports de domination qui structurent non seulement les différents espaces par lesquels elles transitent, le logement, le travail, mais aussi les relations entre compatriotes et avec la population locale aux yeux de laquelle elles deviennent 'l'étrangère'. Comme les autres, Olga désire parfois baisser les bras, rentrer chez elle. Mais elle reste, elle s'accroche. Quelles que soient les configurations migratoires, elles semblent introduire une transformation $\mathrm{du}$ sujet en forme de non-retour. Les trames assujettissantes guident leurs trajectoires en Argentine en fonction d'identités (souvent erronément) attribuées (de femmes, étrangères latinoaméricaines, donc pauvres et peu cultivées). Mais Olga - qui reprend certaines de ces assignations à son compte - ne demeure pas passive, elle se démène, pose ses propres jalons pour faire venir sa famille, décrocher des papiers en règle, organiser à la fois son travail et sa vie familiale, et construire de la stabilité et de la prévisibilité, c'est-à-dire un temps de projection pour soi et les siens.

\section{Domesticité et engagement associatif}

Mais, à 50 ans, le corps d'Olga vient lui imposer des limites. Suite à une première opération, il lui est interdit de porter du poids. Puis on lui diagnostique une arthrose importante de la colonne vertébrale, elle est censée ne plus travailler dans les 
services aux personnes. Son mari lui demande de cesser toute activité de travail, elle accepte cependant une place d'employée domestique chez le fils d'une de ses vieilles protégées, décédée peu de temps avant. Elle travaille quelques heures par jour et ne doit pas faire d'effort physique. Mais c'est moins à ce travail qu'elle consacre son énergie qu'à d'autres causes. Une fois qu'elle cesse toute activité à demeure, Olga reprend ce qu'elle appelle des activités politiques non partisanes. Elle entend par là divers engagements auprès de la communauté péruvienne: elle est vice-présidente puis secrétaire d'une association de migrantes péruviennes, présidente du Conseil de consultations ${ }^{16}$ lié à l'ambassade de son pays, et ambassadrice pour la paix pour l'Universal Peace Federation. Elle regarde en arrière et conclut :

Je viens de là-bas. Ce n'est pas que je suis venue ici [en Argentine], je m'y suis réalisée et je m'y suis plu. Non. Je viens de là-bas et j'ai trouvé ma place. Je ne suis plus la petite fille qui a travaillé à huit ans. Elle a fait son chemin. Ça aurait pu être un autre chemin $[\ldots]$ un chemin sans issue.

Tel un avertissement adressé à la chercheuse, Olga rappelle que sa vie ne débute pas soudainement avec son arrivée en Argentine. À la lumière de ce présent qu'elle revendique ( «j’ai trouvé ma place »), la fillette Olga était certes victime, mais déjà indocile. De cette même indocilité qu'elle se reconnaît encore. Sa vie fut-elle plus gratifiante lorsqu'elle occupait des activités politiques au Pérou, c'est en Argentine, assignée aux secteurs dévalorisés de la domesticité et du care, qu'elle dit sans fatalité avoir trouvé sa place. Aurait-elle réduit ses prétentions ? Ce n'est pas la bonne question. La vie passe et il n'y a pas de retour en arrière, elle doit donc composer avec sa propre réalité. Les dominations qui, en Argentine, s'exercent sur la trajectoire d'Olga et la structurent sont aussi puissantes qu'incontestables; elles sont elles aussi constitutives du sujet Olga. Même ses engagements auprès de la collectivité péruvienne pourraient être lus comme étant au final balisés par ces mêmes rapports sociaux. Mais Olga est aussi ce sujet qui s'en dégage et pose obstinément ses

\footnotetext{
${ }^{16}$ Les Conseils de consultations sont censés représenter la société civile péruvienne vivant à l'étranger. En lien avec les autorités consulaires, ils visent à faciliter la vie des membres de la communauté péruvienne et à œuvrer à la recherche de solutions aux problèmes rencontrés dans le pays d'accueil.
} 
propres marques, qui vient d'une histoire qui déborde l'instantané, le situe et lui donne un sens qui fait sens, et rend sinon caduque, du moins insuffisante, une analyse en termes de domination/consentement.

\section{La trajectoire, analyseur et témoin des ambivalences}

Toutes les femmes rencontrées sont venues en Argentine pour y travailler. Quel que soit leur niveau d'étude et de formation, elles se sont retrouvées dans des 'niches' réservées - emplois domestiques, du care, ou petite vente informelle et pour la plupart, elles y demeurent. À l'instar d'Olga, elles sont plusieurs à occuper en Argentine une position sociale qu'elles n'auraient imaginé pouvoir occuper dans leur pays. Ainsi, lorsqu'elle était enfant, Lucía et sa sœur ont appris à faire le ménage grâce à leur grand-mère pour qui :

Celui qui ne sait pas nettoyer ne sait pas commander. [...] Comment, lorsque vous aurez une employée, saurez-vous commander si vous-même ne savez pas vous y prendre?

Olga est une petite femme énergique, plutôt autoritaire. Son premier emploi fut de domestique, le dernier aussi, pourtant les significations que revêt chacune de ces expériences, à chaque extrémité de sa trajectoire hétérogène, diffèrent du tout au tout. Comme les autres migrantes rencontrées, le retour au pays est de moins en moins sûr, sinon d'ores et déjà exclu. Notre attention a, jusqu'à présent, essentiellement porté sur leur vécu du travail du care à demeure, dont les contraintes physiques et psychologiques sont de nature à briser toute 'entièreté' de soi («l'impression d'être un insecte» selon Rosalba). Outre cela, la fragilité de leur situation se retrouve au niveau du travail et des emplois, des logements, des papiers ${ }^{17}$, de l'accès aux soins, de l'accès à une pension, etc. Il est donc loin de notre propos de

\footnotetext{
${ }^{17}$ En 2003, la loi migratoire 25.871 (réglementée en 2010) déroge la loi Videla, à la fois contraignante et criminalisante. Par cette nouvelle loi et le programme de régularisation Patria Grande (2005), l'Argentine reconnaît le droit des personnes à migrer et la libre-circulation des membres du Mercosur (Le Gall, Sassone 2007). Pour une vision critique des nouvelles formes de contrôle migratoire, voir Domenech (2013). La plupart des enquêtées ont passé dix ou quinze ans sans documents légaux jusqu'au milieu des années 2000.
} 
minimiser ces contraintes ainsi que leurs effets sur des formes de protection de soi au quotidien. Ce qui nous importe, c'est de montrer qu'aucune de ces trajectoires ne se réduit à 'ça' et qu'une situation objectivement dominée peut représenter à la fois une contrainte et (ou) un affranchissement pour le sujet, pour qui elle est 'située' dans son histoire et sa construction personnelle ${ }^{18}$.

L'emploi de domestique actuellement occupé par Olga est lu à travers les multiples changements éprouvés au cours de son histoire, changements qui ont touché sa vie personnelle, professionnelle, amoureuse, publique. Les faits, les actions, les changements de statut, sont moins porteurs de signification en soi que replacés dans des contextes à la fois personnels et historiques, ici et là-bas, le long d'un temps qui fait son œuvre. La lecture portée par les sujets est elle-même travaillée en fonction d'un grand nombre de facteurs : souvenirs, oublis, émotions, interprétations, dénis, défenses. - individu produit par une histoire dont il cherche à devenir le sujet (de Gaulejac 1999), pour ne pas choir dans ce 'chemin sans issue' qui, d'après Olga, menaçait la fillette qu'elle était. En même temps, les descriptions qu'elle offre des contraintes qui pesaient sur l'enfant (délaissée, mise au travail, violentée - sauf par l'oncle qu'elle ne suivra pourtant pas), et jeune adulte (enfermée, 'pondeuse', tenue dans l'ignorance) diffèrent de celles qui ont marqué sa trajectoire postérieure au Pérou (dans les mondes essentiellement masculins du travail et de l'engagement public) et surtout en Argentine (étrangère, isolée, séparée de sa famille, non reconnue dans son travail) jusqu'à ce qu'elle parvienne à construire un certain équilibre personnel, familial et professionnel. Quelles qu'aient pu être les difficultés, elle a essayé de les affronter en tant que sujet de son histoire, fut-ce au prix de compromis avec la réalité et ses propres perceptions. Au niveau du récit rétrospectif, la construction de ce sujet, qui tout à la fois est issu et se dégage des contraintes assujettissantes, prime plutôt que sa position défavorable au prisme des rapports sociaux de sexe, classe, 'race'. Elle est une femme qui s'est dégagée de la violence conjugale, une

\footnotetext{
${ }^{18}$ Cf. la trajectoire de Myriam (Le Quentrec 2009).
} 
Péruvienne qui a su se construire sa place, une personne qui a tout perdu et s'est refait une situation avec sa famille.

Olga est une forte tête qui ne cache pas qu'elle aime jouer les premiers rôles. Son récit témoigne à la fois de contraintes biographiques et de capacités à s'imposer, voire même à soutenir un engagement militant (Polletta 1998). Certaines trajectoires sont davantage que la sienne marquées par la succession de positions fortement structurées par le fait qu'elles sont des femmes, pauvres, ou étrangères. Mais le détail des récits et de la reconstruction des trajectoires témoigne de choix et de ruptures, même de petites transgressions ou de regards critiques, qui les dévient de tout chemin tracé. Point de mécanique absolument parfaite entre les individu.e's et les contraintes qui les assujettissent. Olga est employée domestique 'externe' dans des conditions qui lui conviennent et lui permettent de se consacrer à d'autres activités par lesquelles à la fois elle se reconnaît pleinement et exerce un certain pouvoir sur les autres. Dans d'autres cas cependant (Rosalba, Estrella), même lorsque les conditions assujettissantes de travail et de vie ne permettent aucune projection, les travailleuses posent un regard critique sur leurs situations passées et présentes, ni complètement lucide, ni complètement aveugle, réfléchi et parfois même cruellement juste. Enfin, il apparaît aussi que l'engagement social n'est pas en soi gage 'd'émancipation'. On peut, comme Lucía, être à la fois plus 'émancipée' par la responsabilité qu'implique l'investissement associatif et 'assujettie' par des obligations domestiques (par exemple, la garde prolongée des petits-enfants) ou religieuses.

\section{L'irréductibilité de la vie aux rapports de domination}

Les niveaux micro et macrosociaux interagissent de manière dynamique et changeante au long de la vie. Les trajectoires et représentations témoignent de rapports souvent ambivalents et inachevés aux dominations sociales et produisent une image plus nuancée de sujets qui la subissent et s'en dégagent, la reproduisent et la contestent. L'analyse microsociale est essentielle pour ce qu'elle révèle des écarts à la domination et des méandres dans lesquels les travailleuses inscrivent leurs parcours et leurs discours (Borgeaud-Garciandía 2009 ; Mozère 2010). On peut 
être à la fois, ou tour à tour, 'dominant' et 'dominé', et ces possibilités peuvent se manifester simultanément et varier au cours de l'existence. Le temps long de la trajectoire permet d'en dégager les continuités, les inflexions ; les récits à la première personne du singulier d'en mesurer les bémols, les subtilités, les rugosités.

La migration est extrêmement riche pour l'analyse, en ceci qu'elle introduit du contraste et qu'elle s'avère potentiellement disruptive. Elle permet de mieux mettre au jour ce qui, dans d'autres trajectoires, s'avère moins visible. Elle ne représente pas une 'simple expérience' dans la vie. Point d'inflexion de la trajectoire, elle apparaît comme une 'instance' de resignification des étapes de son existence, de son passé, de son présent et des désirs et perspectives futures. Dans ce sens, il apparaît que les idées de 'trajectoires ascendantes' ou 'descendantes', ou encore l'étiquette 'dominé' à la croisée des différents rapports sociaux ne parvient pas à faire justice des transformations, fluctuations, inflexions, bref de la non-linéarité des trajectoires — et de la lecture qu'en offrent les personnes concernées. Ainsi, l'analyse globale de la structuration des rapports sociaux se complète par une analyse diachronique et synchronique du dynamisme plus 'souterrain', mais riche et potentiellement créateur, de ces rapports au niveau de la vie des gens.

\section{Références}

Attias-Donfut Claudine, Delcroix Catherine (2004). « Femmes immigrées face à la retraite ». Retraite et société, $\mathrm{n}^{\circ} 43$.

Benencia Roberto (2004). "La existencia de modelos históricos contrapuestos en la integración de los migrantes en la sociedad argentina". Les Cahiers ALHIM, n 9 : http://alhim.revues.org/430

Borgeaud-Garciandía Natacha (2008a). Les sujets du labeur. Travail à l'usine, travail de soi et subjectivité des ouvrières et des ouvriers des maquilas du Nicaragua. Thèse de Doctorat, Université PanthéonSorbonne. Lille, Atelier national de reproduction des thèses.

- (2008b). « De la révolution sandiniste au néolibéralisme : l'histoire nicaraguayenne à travers des récits ouvriers ». Nuevo Mundo / Mundos Nuevos : http://nuevomundo.revues.org/index41123.html - (2009). Dans les failles de la domination. Paris, Puf. 
— (2012a). «De l'usine à l'intime. Mise au travail par la maquila et vies d'ouvrières ». Les mondes du travail, $\mathrm{n}^{\circ} 11$.

- (2012b). «Le care à demeure. Le travail des cuidadoras migrantes à Buenos Aires ». Travailler, $\mathrm{n}^{\circ} 28$.

- (2014). «Relations de care, affects et dominations. Le care à demeure à Buenos Aires ». Revue des sciences sociales, ${ }^{\circ} 52$.

Cacopardo María Cristina (2005). "Acerca de las mujeres migrantes en la Argentina: tendencias y mercado de trabajo". In Migraciones, globalización y género en Argentina y Chile. Buenos Aires, CECYM.

Calvelo Laura (2012). "La migración internacional en Argentina hacia 2010". REMHU : Revista interdisciplinar da mobilidade humana, vol. $20, \mathrm{n}^{\circ} 39$.

Castillo Julia, Gurrieri Jorge (2012). "El panorama de las migraciones limítrofes y del Perú en la Argentina a inicios del siglo XXI". In OIM. El impacto de las migraciones en Argentina. Cuadernos Migratorios, $\mathrm{n}^{\circ} 2$.

Cerruti Marcela (2005). "La migración peruana a la Ciudad de Buenos Aires: su evolución y características". Población de Buenos Aires, vol. $2, \mathrm{n}^{\circ} 2$.

- (2009), "Gender and Intra-Regional Migration in South America". Research Paper, ${ }^{\circ} 12$, UNDP, Human Development Reports.

Cortés Rosalia, Groisman Fernando (2004). "Migraciones, mercado de trabajo y pobreza en el Gran Buenos Aires". Revista de la CEPAL, $\mathrm{n}^{\circ} 82$.

Courtis Corina, Pacecca María Inés (2008). Inmigración contemporánea en Argentina: dinámicas y políticas. CEPAL - CELADE, Serie Población y Desarrollo, $\mathrm{n}^{\circ} 84$.

- (2010). "Género y trayectoria migratoria: mujeres migrantes y trabajo doméstico en el Área Metropolitana de Buenos Aires". Papeles de Población, vol. 16, $\mathrm{n}^{\circ} 63$.

Crivelli Karina (2014). "Travailler dans la rue à Buenos Aires. Expériences quotidiennes des vendeuses ambulantes boliviennes ». Revue Tiers Monde, $\mathrm{n}^{\circ} 217$.

Domenech Eduardo (2013). “Las migraciones son como el agua': Hacia la instauración de políticas de "control con rostro humano". Polis, $\mathrm{n}^{\circ} 35$ : http://polis.revues.org/9280

Falquet Jules, Hirata Helena, Kergoat Danièle, Labari Brahim, Le Feuvre Nicky, Sow Fatou (eds) (2010). Le sexe de la mondialisation. Genre, classe, race et nouvelle division du travail. Paris, Presses de Sciences Po. 
Falquet Jules, Lada Emmanuelle, Rabaud Aude (eds) (2006). " (Ré)articulation des rapports sociaux de sexe, classe et 'race' ". Les Cahiers du CEFREF, $\mathrm{n}^{\circ} 14$.

Falquet Jules, Rabaud Aude (2008). «Femmes, genre, migrations et mondialisation ». Les Cahiers du CEDREF, $\mathrm{n}^{\circ} 16$.

Figueroa Blanca (2007). “¿Es como de la familia? Trabajo en servicio doméstico en el Perú". Interculturalidad:

$\mathrm{http}$ //interculturalidad.org/numero04/docs/03c01-Es_como_de_ la_familia-Figueroa.Blanca.pdf

Galloro Piero-D., Pascutto Tamara, Serré Alexia (2010). « De l'immigré à l'émigré ? L'entretien biographique en contexte(s) migratoire(s) ». Temporalités, $\mathrm{n}^{\circ} 11$ : http://temporalites.revues.org/1168

Gaulejac (de) Vincent (1999). L'histoire en héritage : roman familial et trajectoires sociales. Paris, Desclée de Brouwer.

Kergoat Danièle (2009). «Dynamique et consubstantialité des rapports sociaux ». In Dorlin Elsa (ed). Sexe, race, classe. Pour une épistémologie de la domination. Paris, Puf.

Kergoat Danièle, Miranda Adelina, Ouali Nouria (eds) (2011). « Migrantes et mobilisées ». Cahiers du genre, $\mathrm{n}^{\circ} 51$.

Le Gall Julie, Sassone Susana María (2007). « Tournant des politiques migratoires en Argentine. Vers une nouvelle politique territoriale? » EchoGéo, $\mathrm{n}^{\circ} 3$ : http://echogeo.revues.org/1850

Le Quentrec Yannick (2009). « Myriam la militante : entre domination et puissance d'agir ». In Cardon Philippe, Kergoat Danièle, Pfefferkorn Roland (eds). Chemins de l'émancipation et rapports sociaux de sexe. Paris, La Dispute « Le genre du monde».

Montvalon (de) Prune (2013). «Trans, migrantes et prostituées : dominations imbriquées et espaces de négociation ». Cahiers de l'Urmis, n 14 : http://urmis.revues.org/1196

Moujoud Nasima (2008). «Effets de la migration sur les femmes et sur les rapports sociaux de sexe. Au-delà des visions binaires ». Les cahiers du CEDREF, $\mathrm{n}^{\circ} 16$.

Mozère Liane (2010). «La mondialisation comme arène de "trouvailles accumulées'? Des domestiques philippines à Paris ». In Falquet Jules, Hirata Helena, Kergoat Danièle et al. (eds).

Pacecca María Inés (2009). "La migración boliviana, peruana y paraguaya a la Argentina (1980-2005)". Congress of the Latin American Association, Rio de Janeiro, Brésil.

Pfefferkorn Roland (2012). Genre et rapports sociaux de sexe. Lausanne, Page deux. 
Polletta Francesca (1998). "Contending Stories: Narrative in Social Movements". Qualitative Sociology, vol. 21, n 4.

Zeneidi Djemila (2013). Femmes / fraises. Import / export, Paris, Puf. 